\title{
Article
}

\section{W-Band Modular Antenna/Detector Array for the Electron Cyclotron Emission Imaging System in KSTAR}

\author{
Gwan Hui Lee ${ }^{1}$, Jung Seok Lee ${ }^{2}$, Dong Hwi Kim ${ }^{2}$, Syifa Haunan Nashuha ${ }^{2}$, Mun Ju Kim ${ }^{2}$, Byung Cheol Min ${ }^{2}$, \\ Jae Hyun Lee ${ }^{3}$, Woo Chang Lee ${ }^{3}$, Gun Su Yun ${ }^{4}$ (D), Tae Gyu Kim ${ }^{5}$, Bong Tae Kim ${ }^{6}$, Hyun Chul Choi ${ }^{2}$, \\ Hyeon Keo Park ${ }^{1}$, Yong Kyoon In ${ }^{1}$ and Kang Wook Kim ${ }^{2}$,*
}

Citation: Lee, G.H.; Lee, J.S.; Kim, D.H.; Nashuha, S.H.; Kim, M.J.; Min, B.C.; Lee, J.H.; Lee, W.C.; Yun, G.S.; Kim, T.G.; et al. W-Band Modular Antenna/Detector Array for the Electron Cyclotron Emission Imaging System in KSTAR. Appl. Sci. 2022, 12, 2431. https://doi.org/10.3390/ app12052431

Academic Editors: Ernesto Limiti, Atsushi Mase and Neville C. Luhmann

Received: 19 January 2022 Accepted: 23 February 2022 Published: 25 February 2022

Publisher's Note: MDPI stays neutral with regard to jurisdictional claims in published maps and institutional affiliations.

Copyright: (C) 2022 by the authors. Licensee MDPI, Basel, Switzerland. This article is an open access article distributed under the terms and conditions of the Creative Commons Attribution (CC BY) license (https:// creativecommons.org/licenses/by/ $4.0 /)$.
1 Ulsan National Institute of Science and Technology, Ulsan 44919, Korea; gwanhuilee@unist.ac.kr (G.H.L.); hyeonpark@unist.ac.kr (H.K.P.); inyongkyoon@unist.ac.kr (Y.K.I.)

2 School of Electronic and Electrical Engineering, Kyungpook National University, Daegu 41566, Korea; j.seok1020@gmail.com (J.S.L.); eastsine766@gmail.com (D.H.K.); nsh11@knu.ac.kr (S.H.N.); dranswn@knu.ac.kr (M.J.K.); minbc4658@knu.ac.kr (B.C.M.); hcchoi@ee.knu.ac.kr (H.C.C.)

3 Korea Institute of Fusion Energy, Daejeon 34133, Korea; jaehyun@kfe.re.kr (J.H.L.); wclee@kfe.re.kr (W.C.L.)

4 Department of Physics, Pohang University of Science and Technology, Pohang 37673, Korea; gunsu@postech.ac.kr

5 EM-wise Communications Co., Daegu 41566, Korea; ktg21@em-wise.com

$6 \quad$ PEOPLEWORKS Inc., Gumi 39376, Korea; galleryway@peopleworks.co.kr

* Correspondence: kang_kim@ee.knu.ac.kr

\begin{abstract}
A design of a modular antenna/detector array for the electron cyclotron emission (ECE) imaging system at the Korea Superconducting Tokamak Advanced Research (KSTAR) is proposed. The modular antenna/detector array is based on a unit antenna/detector module, which consists of an elliptical mini-lens, a dual-dipole antenna, an antenna balun, a low-noise amplifier, and a metal frame. The proposed modular antenna/detector array resolves the problem in the conventional antenna/detector array where one faulty channel requires the entire array to be removed for the service. With the proposed modular array, each channel module can be easily and independently removed and replaced without interference to the rest of the array, thus minimizing the interrupted service time for maintenance. Moreover, the unit channel modules can be efficiently updated under a variety of the tokamak operation conditions. The antenna/detector modules are optimized to have improved performance, and are tested in a W-band test setup, and consistently provide the gain increase by 10 20 dB as compared with the conventional antenna/detector array. A set of the proposed modular antenna/detector array is currently installed and tested in the KSTAR ECE imaging system, and will consistently produce the improved ECE imaging to monitor MHD instability activities under various plasma operation conditions.
\end{abstract}

Keywords: modular antenna/detector array; elliptical mini-lens; dual-dipole antenna; wideband antenna balun; maintenance; W-band; ECE; KSTAR

\section{Introduction}

Thermonuclear fusion technology has been actively explored for generating safe, abundant, and radioactive-free green energy. Among various candidates of fusion energy generating machines, a tokamak, which is a magnetic confinement device, is the most promising and most widely investigated [1]. For stable operation of the tokamak, the magneto-hydrodynamics (MHD) instabilities such as tearing mode, edge localized mode, and sawtooth, need to be fully understood and controlled [2-5]. Therefore, in order to observe and analyze the MHD instabilities in detail, various versions of the electron cyclotron emission (ECE) imaging system, which monitors the plasma temperature fluctuations, are utilized in major tokamak devices (e.g., KSTAR, WEST, EAST, DIII-D) [6-11].

In a tokamak, strong magnetic fields are used to confine a high-temperature plasma column, and the charged particles gyrate with a frequency proportional to the strength 
of the magnetic field. Thus, the electron cyclotron emission radiation is generated by the rotation of electrons following along the magnetic field lines. In addition, the rotating electrons radiate the harmonics of the ECE frequencies. Since the radiation from an optically thick plasma is close to the blackbody radiation, the intensity of the ECE radiation is assumed to be proportional to the electron temperature of the plasma. Furthermore, each ECE radiation frequency represents the radial position of the corresponding magnetic field. Therefore, the intensity measurement of the ECE radiation in various frequency bands allows for the identification of the radially localized electron temperature fluctuation $[12,13]$.

In a tokamak plasma, an ECE measurement can be performed for an ordinary mode (O-mode) or an extraordinary mode (X-mode). In the Korea Superconducting Tokamak Advanced Research (KSTAR), with the high magnetic field $\left(B_{0}=2 \mathrm{~T}\right)$ condition, most of the outward propagated radiation is the second harmonic of the ECE frequency in the X-mode waves, typically lying in W/F-band $(75 \sim 140 \mathrm{GHz})$. With an even higher magnetic field $\left(B_{0}=3 \mathrm{~T}\right)$, the $\mathrm{O}$-mode radiation occurs in $75 \sim 120 \mathrm{GHz}$, and the $\mathrm{X}$-mode radiation occurs in 120 140 GHz [14].

The ECE imaging system measures 2D images of the electron temperature fluctuations according to radial and vertical points of the tokamak plasma. Additionally, in KSTAR, the antenna/detector array was designed with various RF components (e.g., antenna, diode, etc.) especially optimized to measure $\mathrm{W}$-band (75 to $110 \mathrm{GHz}$ ) signals out of the wide spectrum of ECE radiated frequencies. A conventional antenna/detector array for the ECE imaging system is shown in Figure 1. The antenna/detector array consisted of 12 channels to receive the vertical ECE signals from the tokamak. Each channel was comprised of an elliptical mini-lens and an antenna/detector. The 12 sets of antennas/detectors were printed conjointly on a single board. Additionally, the 12 elliptical mini-lenses were fabricated in a single block structure. Therefore, in case of failure or degraded performance even with a single channel, the entire array module had to be removed for the repair service. This caused the maintenance process to be costly and time-consuming with the conventional array.

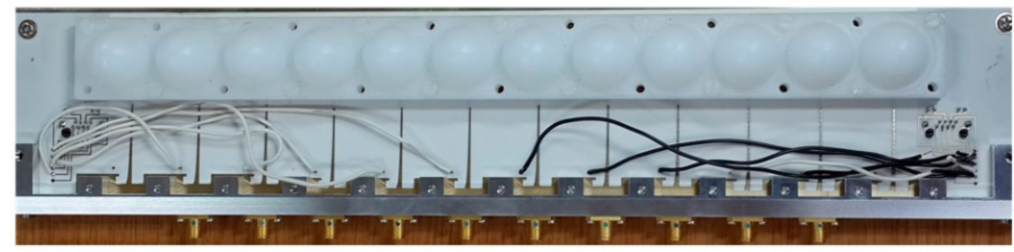

(a)

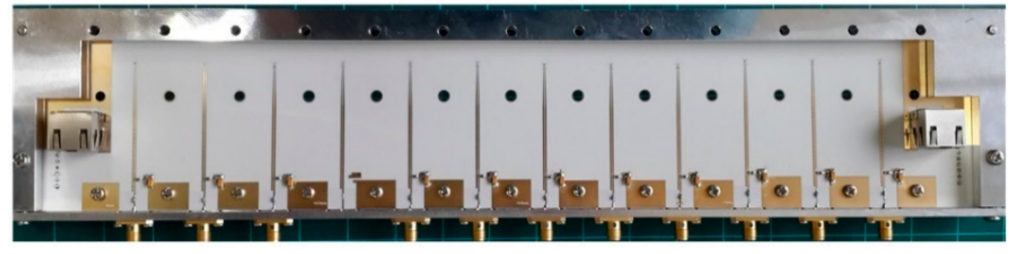

(b)

Figure 1. Conventional antenna/detector array for the KSTAR ECE imaging system: (a) top view, (b) bottom view.

While various conventional ECE imaging systems have been actively utilized in tokamaks around the world, there are strong needs for improved systems for more effective diagnostic operations [15-18]. Recently, a new heterodyne W-Band ECE imaging system on DIII-D, which could dramatically improve signal-to-noise ratio, was presented in [19]. However, the system showed a non-flat conversion gain (33-47 dB), meaning that the frequency range of the system would be limited. The heterodyne ECE receiver requires ultra-wideband MMICs and microwave/mm-wave components to cover the full frequency band of ECE measurements ( 10s of GHz). When commercial MMICs for the required band are not available, customized MMICs should be developed. Additionally, the uniform 
and precise assembly for the whole receiver array may be very expensive. Therefore, a conventional-type ECE imaging system, which uses an array of a dielectric lens integrated with an antenna and a Schottky diode, may still serve as a practical ECE imaging system for most tokamak devices as long as the intensity of the plasma-radiated ECE signal is not so weak for the measurements. Additionally, continued efforts are expected to improve the performance and flexibility of the ECE imaging system.

In this paper, a new design of a modular antenna/detector array, based on a unit antenna/detector module, for the KSTAR ECE imaging system is presented for efficient maintenance and performance improvement. Each unit antenna/detector module, which is mounted in an array frame, is composed of an elliptical mini-lens, a dual-dipole antenna, a Schottky diode, an ultra-wideband balun, a low-noise amplifier (LNA), and a unit frame.

The dual-dipole antenna and elliptical mini-lens are optimized for W-band ECE signals [20]. Wideband, high-gain performance can be achieved by combining the antenna with the elliptical dielectric lens since the dielectric lens helps in focusing the ECE signals to the center of the dual-dipole antenna, where a Schottky diode is placed. The W-band ECE signal is mixed with a W-band local oscillator (LO) signal by the Schottky diode, generating an intermediate frequency (IF) signal. The IF signal propagates through an ultra-wideband balun consisting of a coplanar strip (CPS) taper and a CPS-to-microstrip line (MSL) transition. Additionally, an LNA is integrated to amplify the weak IF signal from the ECE radiation. For efficient maintenance of the ECE imaging system, each unit antenna/detector module can be easily and independently removed and replaced on the array frame. Moreover, the bias lines for the Schottky diode and amplifier are connected from the bias PCBs to each unit module using a connector socket. The performance of each unit antenna/detector module is also optimized to have higher gain and more flat frequency response as compared with the conventional module. This study is the first published work that discusses in detail about the improved antenna/detector array for the ECE imaging system at KSTAR.

\section{KSTAR ECE Imaging System}

A block diagram of the W-band ECE imaging system installed at KSTAR is shown in Figure 2. The ECE signals emitted from each radially localized point at the tokamak plasma pass through a series of optical lenses to form parallel Gaussian beams, which radiate uniformly upon the elliptical mini-lenses attached to the antenna/detectors [14]. For the polarization alignment and filtering of the incoming ECE signals, three FSS (frequency selective surface) layers (a half-wave plate, notch filter, and dichroic filter) are placed in front of the array box. In details, the half-wave plate rotates the polarization of the incident signal by $90^{\circ}$ (used only for the O-mode wave), the notch filter is used to block the strayed ECE heating signals, and the dichroic filter works to block low-frequency components. Then, the plasma-radiated ECE signal impinge upon a tilted beam splitter, which divides the signal into two paths. The tilted beam splitter also allows a perpendicularly fed LO signal to be split so that the ECE (RF) and LO signals are spatially combined at each path. These RF-LO combined signals at each path radiate uniformly over the 12 elliptical minilenses attached to the dual-dipole antennas. Then, each combined RF-LO signal, passing through the elliptical mini-lens and dual dipole antenna, is mixed in a Schottky diode, which is located between the two dipole radiators, to produce an IF signal with a frequency range of 2 to $9 \mathrm{GHz}$. 


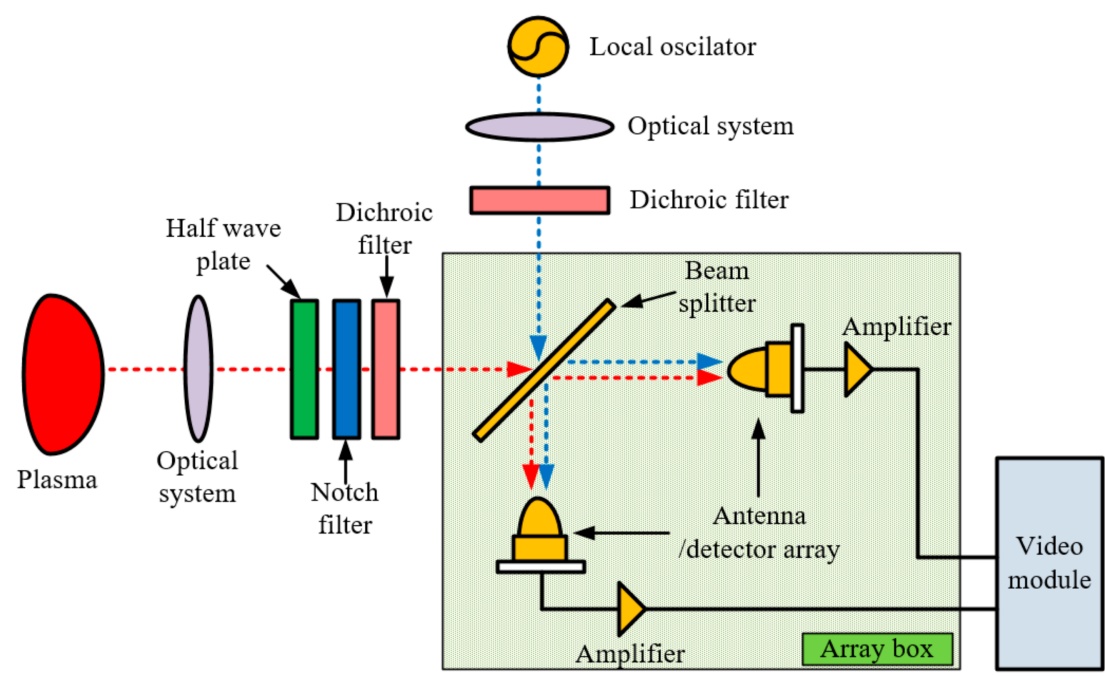

Figure 2. Block diagram of the KSTAR ECE imaging system.

Using an external electronic module (video module), this IF signal is split into 8 frequency channels, and each channel is down-converted again to produce a video signal to monitor the amplitude and fluctuation characteristics.

\section{Antenna/Detector Array Design}

\subsection{Modular Antenna/Detector Array}

A perspective view of the proposed modular antenna/detector array is shown in Figure 3a. In Figure 3b, an exploded view shows the modular antenna/detector array consisting of an array frame, 12 individually installed unit antenna/detector modules, and a pair of DC bias PCBs (printed circuit board). The array frame, made of aluminum, holds the 12-unit antenna/detector modules and the bias PCBs.

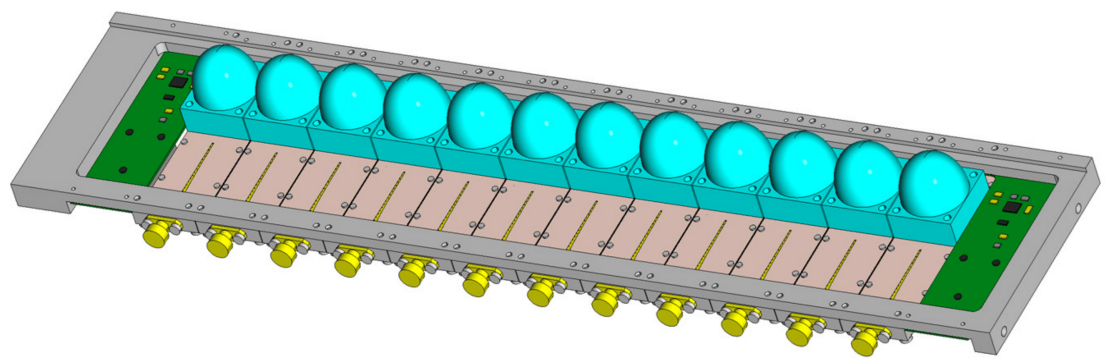

(a)

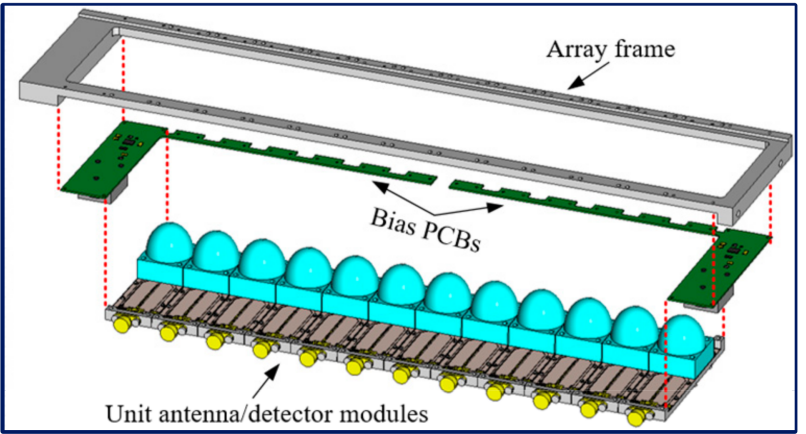

(b)

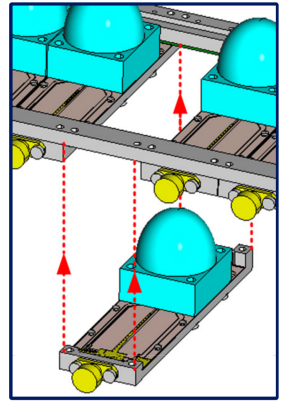

(c)

Figure 3. Proposed modular antenna/detector array: (a) perspective view, (b) exploded view, (c) installation of the unit antenna/detector module.

By adopting the modular design for the antenna/detector array, each unit antenna/ detector module can be attached to or detached from the array frame without interference 
with adjacent channels, as shown in Figure 3c. The bias PCBs (designed with the 40-mil FR-4 substrate) are assembled on the bottom of the array frame. The total size of the proposed antenna/detector array is $425.7 \times 104.5 \times 38.8 \mathrm{~mm}$.

\subsection{Unit Antenna/Detector Module}

A perspective view of the unit antenna/detector module is shown in Figure 4a. The unit antenna/detector module consists of an elliptical mini-lens, a dual-dipole antenna with a Schottky diode, an antenna balun, an LNA, and a unit frame, as shown in Figure 4b. The elliptical mini-lens, made of high-density polyethylene (HDPE) with dielectric constant of 2.27 , is placed at the bottom side of the dual dipole antenna. The dual dipole antenna with an antenna balun is printed on the Rogers 4003 substrate with 12 mil thickness. At the center location of the dual dipole radiators, a Schottky diode (W-Band single anode diode from Virginia Diodes) is mounted. An LNA (HMC772LC4 of Analog Devices) with a gain of $15 \mathrm{~dB}$ and a noise figure of $1.8 \mathrm{~dB}$ is placed at the end of the antenna balun. A bias connector, to supply bias voltages to the LNA and the Schottky diode, is mounted at the upper section on the bottom side of the antenna substrate. The unit frame is manufactured of aluminum. The dimension of the unit antenna/detector module is $27.7 \times 95.0 \times 32.2 \mathrm{~mm}$.

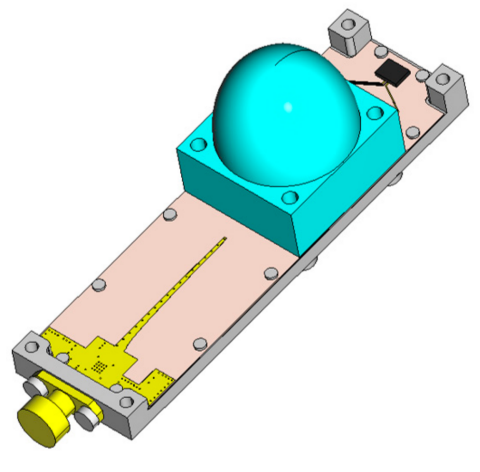

(a)

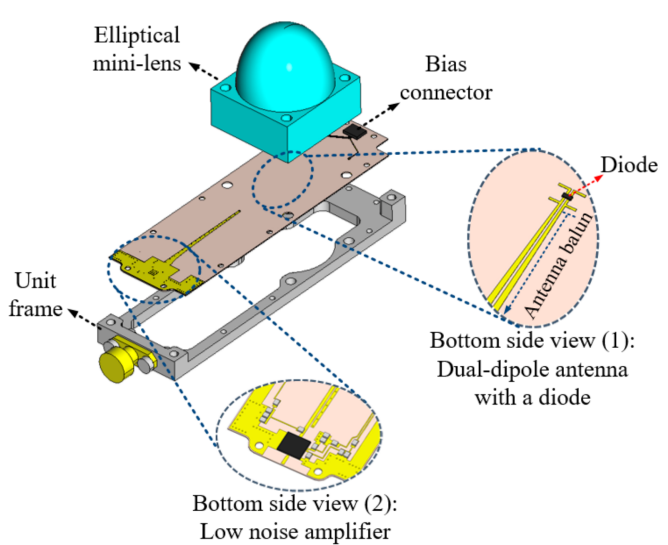

(b)

Figure 4. Unit antenna/detector module: (a) perspective view, (b) exploded view.

\subsubsection{Elliptical Mini-Lens}

A perspective view of the proposed elliptical mini-lens is shown in Figure 5a. The proposed elliptical mini-lens consists of two dielectric structures: a half-elliptical lens and a rectangular dielectric block. The half-elliptical lens is placed on the top of the rectangular dielectric block. The rectangular dielectric block with the dimensions of $l_{x}$ and $l_{y}$ in the $x$ - and $y$-directions is placed on the bottom side of the dual-dipole antenna. The width $l_{y}$ is selected as $27.5 \mathrm{~mm}$, which is larger than twice the minor axis $b$ to prevent interference from nearby channels. Additionally, to accommodate the screw holes, the width $l_{x}$ is selected as $30.0 \mathrm{~mm}$. In Figure $5 \mathrm{~b}$, a cross-sectional view of the proposed dielectric lens in the $x$-z plane is shown. The major axis $a$ and minor axis $b$ are related with the relative dielectric constant $\left(\varepsilon_{r}=2.27\right)$ as

$$
a=b / \sqrt{1-1 / \varepsilon_{r}} .
$$




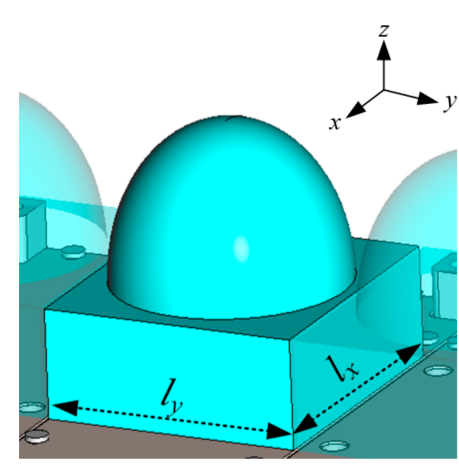

(a)

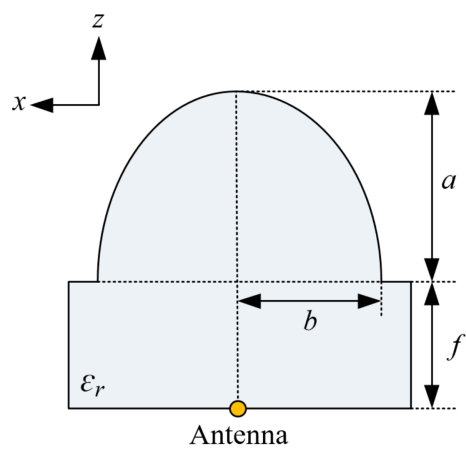

(b)

Figure 5. Elliptical mini-lens: (a) perspective view, (b) side view ( $x-z$ plane).

With consideration of the channel spacing and the focal point size, the minor axis $b$ is chosen as $12.6 \mathrm{~mm}$, and the major axis $a$ of the half-elliptical lens is then calculated as 16.8 $\mathrm{mm}$ [21]. For the collimation of the incident beam towards antenna, the focal point of the half-elliptical lens is located at the bottom of the rectangular dielectric block. Therefore, the height $f$ of the rectangular dielectric block is designed to be equal the focal length, which is calculated as $11.8 \mathrm{~mm}$ from the center of the ellipse using Equation (2) [22].

$$
f=a / \sqrt{\varepsilon_{r}}
$$

\subsubsection{Dual-Dipole Antenna/Detector with an Ultra-Wideband Antenna Balun}

A dual-dipole antenna with a Schottky diode and an antenna balun is illustrated in Figure 6. From $\mathrm{AA}^{\prime}$ to $\mathrm{BB}^{\prime}$, the dual-dipole antenna consists of two dipole radiators and a Schottky diode. The dual-dipole antenna is designed to have the center frequency of $90 \mathrm{GHz}$ [23]. The dimensions of the dual-dipole antenna are $l=0.838 \mathrm{~mm}, d=0.965 \mathrm{~mm}$, $w_{1}=0.152 \mathrm{~mm}$, and $w_{2}=0.127 \mathrm{~mm}$. For the detection of W-band ECE signals, a Schottky diode working up to W/F-band is used. The Schottky diode is mounted at the center location of the dual-dipole radiators to generate an IF signal by mixing the ECE (RF) and LO signals. An external bias voltage is supplied to turn on the Schottky diode with the optimum bias condition.

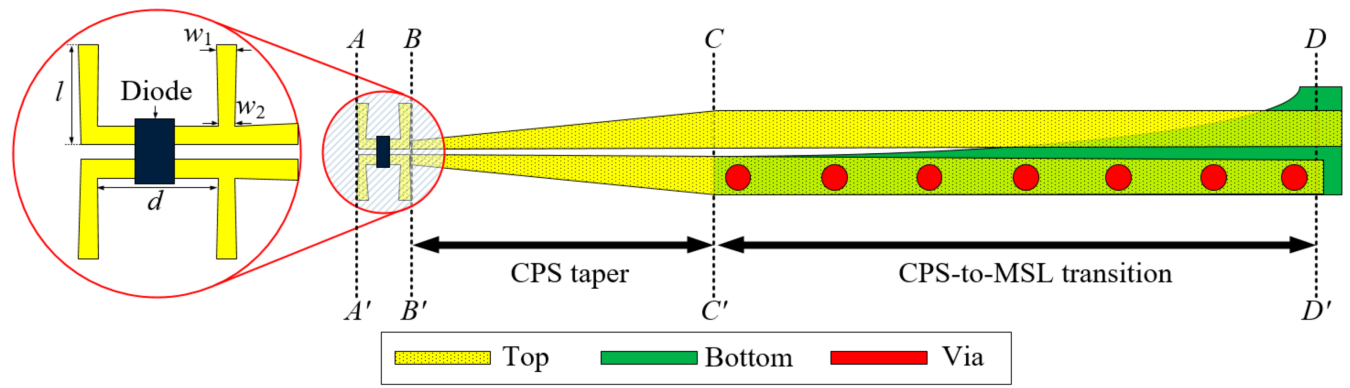

Figure 6. Schematic view of the dual-dipole antenna/detector with a Schottky diode and an antenna balun.

To feed the dual-dipole antenna, an ultra-wideband balun is connected to the CPSbased antenna feeding line. The antenna balun consists of a CPS taper ( $\mathrm{BB}^{\prime}$ to $\left.\mathrm{CC}^{\prime}\right)$ and a CPS-to-MSL transition ( $\mathrm{CC}^{\prime}$ to $\left.\mathrm{DD}^{\prime}\right)$. The CPS linewidth of the dual-dipole antenna feeding point is very narrow $(6 \mathrm{mil})$, resulting in high line impedance $\left(157 \Omega\right.$ at $\left.\mathrm{BB}^{\prime}\right)$. In order to match line impedance for ultra-wideband and low-loss performance, a CPS impedance taper (from $157 \Omega$ at $\mathrm{BB}^{\prime}$ to $115 \Omega$ at $\mathrm{CC}^{\prime}$ ) and a CPS-to-MSL transition (from $115 \Omega$ at $\mathrm{CC}^{\prime}$ to $50 \Omega$ at $\mathrm{DD}^{\prime}$ ) are used. The CPS-to-MSL transition gradually changes the line impedance and field distribution from CPS to MSL by increasing the line width of the bottom ground plane. The line characteristic property of each section of the CPS-to-MSL 
transition is accurately analyzed using the Schwarz-Christoffel transformation with an analytical model [24]. In the analytical model, each section of the CPS-to-MSL transition is divided into three regions, and the capacitances $\left(C_{1}, C_{2}\right.$, and $\left.C_{3}\right)$ of each region are calculated [25]. Hence, the characteristic line impedance of each section is calculated by Equations (3) and (4).

$$
\begin{gathered}
Z_{0}=\frac{120 \pi \varepsilon_{0}}{\sqrt{\varepsilon_{e f f}}\left(C_{1}+C_{2} / \varepsilon_{r}+C_{3}\right)} \\
\varepsilon_{e f f}=\frac{C_{1}+C_{2}+C_{3}}{C_{1}+C_{2} / \varepsilon_{r}+C_{3}}
\end{gathered}
$$

To obtain ultra-wideband performance and to minimize line loss in the presence of a big line impedance difference, the line impedances from the CPS feed (157 $\Omega$ at BB') to MSL (50 $\Omega$ at $\mathrm{DD}^{\prime}$ ) are adjusted to form an optimal Klopfenstein taper [24]. The minimum operating frequency of the transition structure is determined by the length of the transition, and the length of the antenna balun is obtained as $49 \mathrm{~mm}$ to have the minimum operating frequency of $2 \mathrm{GHz}$ [26].

\section{Fabrication and Measurement}

\subsection{Fabrication}

Top and bottom views of the fabricated antenna/detector array are shown in Figure 7a. The unit antenna/detector modules are separately installed in an array frame to form 12 channels. The bias voltages of the unit antenna/detector module (for the diode: $+0.7 \mathrm{~V}$, for the LNA: $+5 \mathrm{~V}$ ) are provided through the bias connectors on the bias PCB. In Figure 7b, a picture of the fabricated unit antenna/detector module is shown. The antenna/detector PCB is attached to the unit frame, and the elliptical mini-lenses are mounted individually on the top side of each unit antenna/detector module using HDPE dielectric screws.

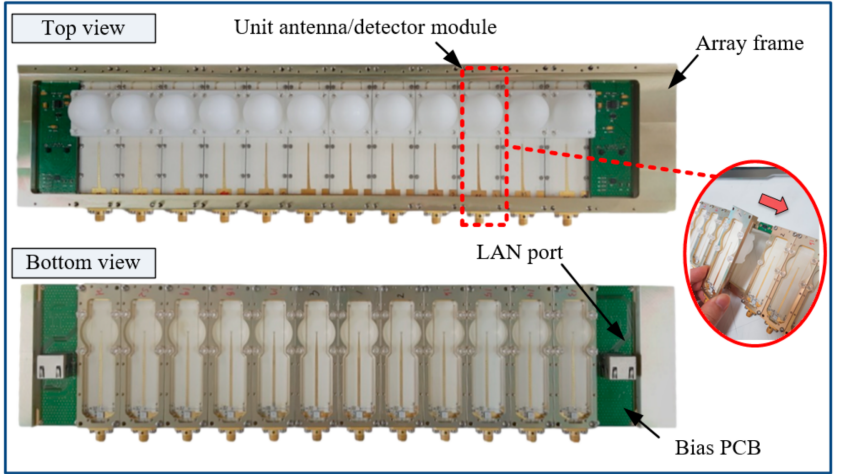

(a)

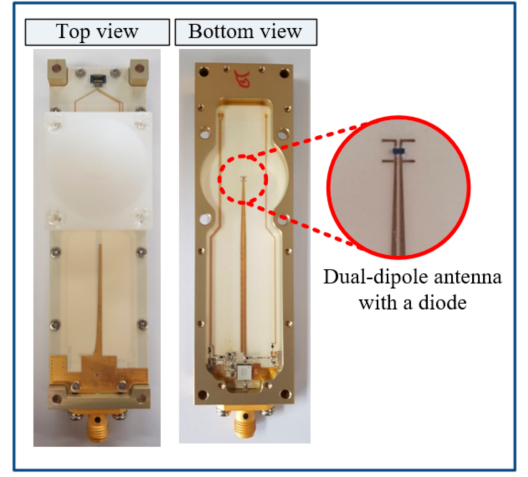

(b)

Figure 7. Fabricated modular antenna/detector array: (a) top and bottom view of the modular antenna/detector array consisting of the unit antenna/detector modules, (b) top and bottom view of the unit antenna/detector module.

\subsection{Measurements}

To measure the performance of the unit antenna/detector module for the KSTAR ECE imaging system, a measurement setup is configured, as shown in Figure 8. Two W-band sources are used as RF and LO sources, each of which consists of a $12.5-18.3 \mathrm{GHz}$ source, a $6 x$ frequency multiplier, a W-band amplifier, and a W-band horn antenna. The RF source (WR10AMC-I of Virginia Diodes) with the output power of $+20 \mathrm{dBm}$ radiates onto a tilted beam splitter. In the perpendicular direction, the LO source (AMC-10-RFHB0 of Millitech) impinges onto the same beam splitter. One path of the combined RF-LO signal propagates towards the modular antenna/detector array. The down-converted IF signal by the Schottky diode passes through the antenna balun, amplified, and then analyzed by the 
spectrum analyzer (Agilent E4407B). The radiation patterns of the unit antenna/detector module are also measured by rotating the unit/antenna detector module from $-30^{\circ}$ to $30^{\circ}$.

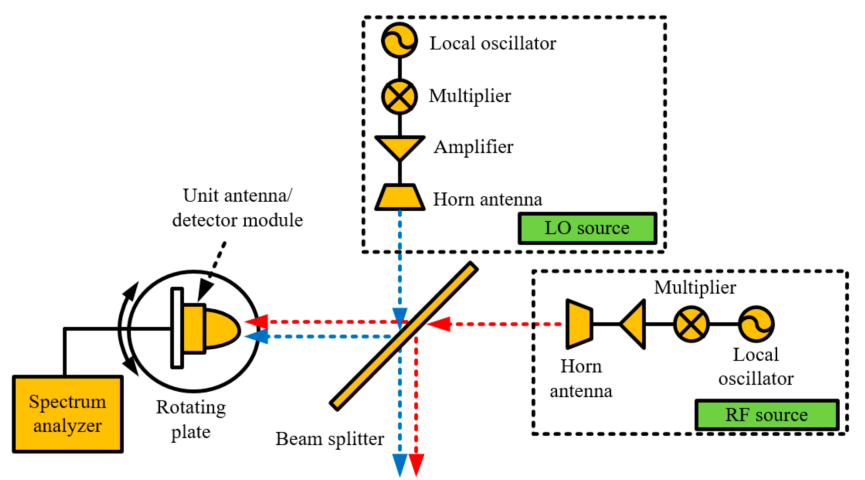

Figure 8. Block diagram of the measurement setup.

The radiation patterns are measured with the test set-up, and the results are shown in Figures 9 and 10. In Figure 9, the measured radiation patterns in the E-plane are presented at RF frequencies of 75, 85, 95, 100, and $105 \mathrm{GHz}$ with IF frequencies of 2 and $9 \mathrm{GHz}$. In Figure 9a, the E-plane radiation patterns at various RF frequencies with the $2 \mathrm{GHz} \mathrm{IF}$ signal are shown. The frequencies of the LO signal are selected as $2 \mathrm{GHz}$ higher than the corresponding RF frequencies. As the RF frequency is increased, the $3 \mathrm{~dB}$ beamwidth is decreased: i.e., $8^{\circ}$ at $75 \mathrm{GHz}, 8^{\circ}$ at $85 \mathrm{GHz}, 6^{\circ}$ at $95 \mathrm{GHz}$, and $5^{\circ}$ at $105 \mathrm{GHz}$. The left and right side-lobe levels are $-22.4 \mathrm{~dB}$ and $-23.3 \mathrm{~dB}$ at $2 \mathrm{GHz}$, respectively. In Figure $9 \mathrm{~b}$, the E-plane radiation patterns are shown for the IF frequency of $9 \mathrm{GHz}$, where the LO frequencies are set to $84,94,104$, and $96 \mathrm{GHz}$. It is observed that, as the RF frequency increased, the $3 \mathrm{~dB}$ beamwidth decreased as $7^{\circ}, 7^{\circ}, 6^{\circ}$, and $5^{\circ}$. The left and right side-lobe levels are $-25.3 \mathrm{~dB}$ and $-21.9 \mathrm{~dB}$ at $9 \mathrm{GHz}$, respectively.

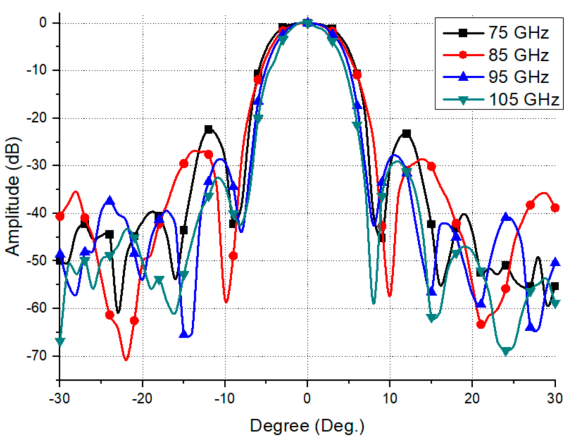

(a)

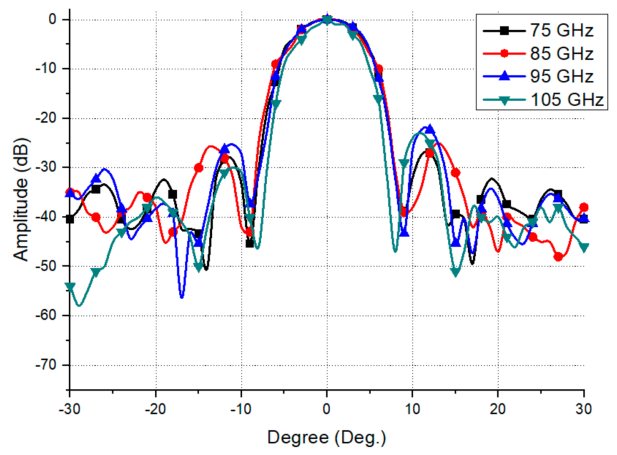

(b)

Figure 9. Measured radiation patterns of the proposed unit antenna/detector module in the E-plane: (a) IF frequency $=2 \mathrm{GHz}$, (b) IF frequency $=9 \mathrm{GHz}$.

Figure 10 exhibits the H-plane radiation patterns with IF frequencies of $2 \mathrm{GHz}$ and $9 \mathrm{GHz}$. With the $2 \mathrm{GHz}$ IF frequency, the frequency of the LO signal is again selected as $2 \mathrm{GHz}$ higher than the RF frequencies. As shown in Figure 10a, the radiation patterns with RF frequencies of 75, 85, 95, and $105 \mathrm{GHz}$ are presented. As RF frequency increases, $3 \mathrm{~dB}$ beamwidth is slightly decreased: i.e., $6^{\circ}$ at $75 \mathrm{GHz}, 6^{\circ}$ at $85 \mathrm{GHz}, 6^{\circ}$ at $95 \mathrm{GHz}$, and $5^{\circ}$ at $105 \mathrm{GHz}$. The left and right side-lobe levels are $-20.2 \mathrm{~dB}$ and $-26.5 \mathrm{~dB}$ at $2 \mathrm{GHz}$, respectively. The left side-lobe level may have been increased due to the interference caused by the signal propagating through the side of the antenna balun. In Figure 10b, the H-plane radiation patterns are shown with the IF frequency of $9 \mathrm{GHz}$. The LO frequencies are selected as 84, 94, 104, and $96 \mathrm{GHz}$. By increasing the frequency, the $3 \mathrm{~dB}$ beamwidth decreased as $7^{\circ}, 6^{\circ}, 5^{\circ}$, and $5^{\circ}$ at $75,85,95$, and $105 \mathrm{GHz}$, respectively. With the IF frequency 
of $9 \mathrm{GHz}$, the left and right side-lobe level are $-18.3 \mathrm{~dB}$ and $-27.4 \mathrm{~dB}$, respectively. In a similar fashion to the radiation patterns with the $2 \mathrm{GHz}$ IF frequency as shown in Figure 10a, all radiation patterns with the $9 \mathrm{GHz}$ IF frequency show asymmetric H-plane patterns, possibly caused by the presence of the antenna balun.

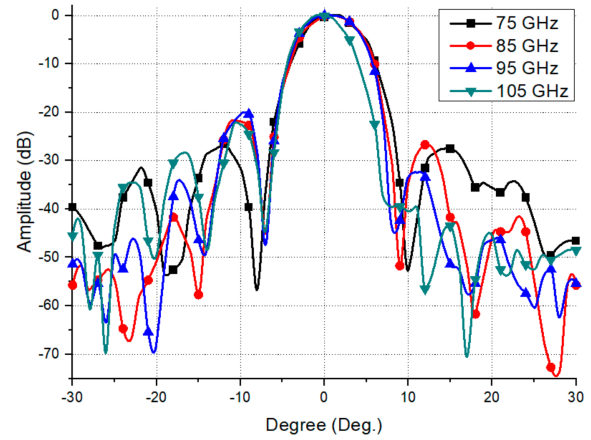

(a)

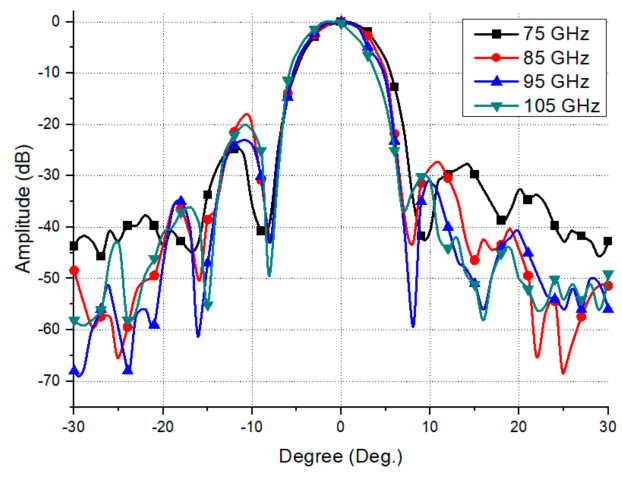

(b)

Figure 10. Measured radiation patterns of the proposed unit antenna/detector module in the H-plane: (a) IF frequency $=2 \mathrm{GHz}$, (b) IF frequency $=9 \mathrm{GHz}$.

In Figure 11, the measured gain increase of the proposed antenna/detector array as compared with the conventional antenna/detector array is presented. In Figure 11a, with an RF frequency range of 75-105 GHz, the gain increases are displayed with two IF frequencies (2 $\mathrm{GHz}$ and $9 \mathrm{GHz}$ ). The proposed antenna/detector array provides the maximum gain increase of $20.8 \mathrm{~dB}$ with the IF frequency of $2 \mathrm{GHz}$, and the minimum gain increase of $9.4 \mathrm{~dB}$ with the IF frequency of $9 \mathrm{GHz}$. It can also be observed that the gain is increased by over $15 \mathrm{~dB}$ for all RF frequencies from $75 \mathrm{GHz}$ to $100 \mathrm{GHz}$. This implies that the obtained gain increase is an aggregate gain of the overall components of the antenna/detector array (e.g., optimized antenna with balun, elliptical mini-lens) in addition to the LNA with $15 \mathrm{~dB}$ gain. However, as $\mathrm{RF}$ frequency increases, the gain increase is deteriorated at frequencies over $100 \mathrm{GHz}$ due to the higher conversion loss of the Schottky diode. Nevertheless, gain increase of over $12 \mathrm{~dB}$ has been achieved at all RF frequencies.

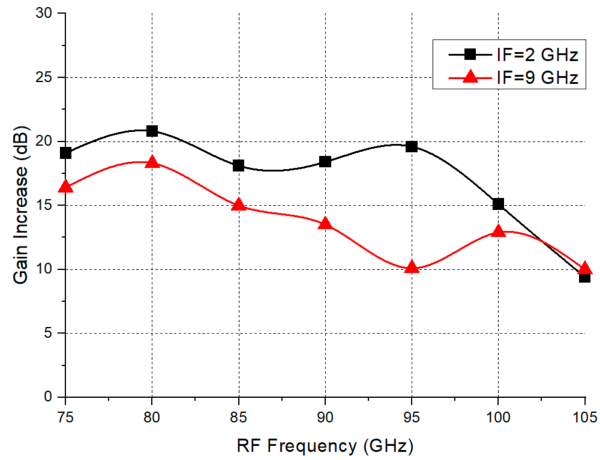

(a)

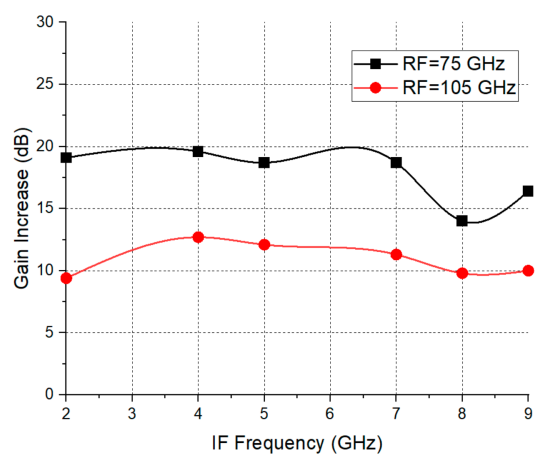

(b)

Figure 11. Measured gain increase of the proposed antenna/detector array as compared with the conventional antenna/detector array: (a) gain increase with IF frequencies of $2 \mathrm{GHz}$ and $9 \mathrm{GHz}$, (b) gain increase with RF frequencies of $75 \mathrm{GHz}$ and $105 \mathrm{GHz}$.

In Figure 11b, with an IF frequency range of $2-9 \mathrm{GHz}$, the gain increases are displayed at two RF frequencies (75 GHz and $105 \mathrm{GHz}$ ). When the RF frequency is set at $75 \mathrm{GHz}$, the gain increase is over $15 \mathrm{~dB}$ for most frequencies with the maximum value of $19.6 \mathrm{~dB}$ and the minimum value of $14 \mathrm{~dB}$. Almost flat gain increases are shown except near the IF frequency of $8 \mathrm{GHz}$. At the RF frequency of $105 \mathrm{GHz}$, the gain increase is under $15 \mathrm{~dB}$ with 
the maximum of $12.7 \mathrm{~dB}$ and the minimum of $9.4 \mathrm{~dB}$. It can be observed that a gain increase of over $\sim 10 \mathrm{~dB}$ as compared with the conventional antenna/detector has been achieved.

\subsection{Proposed Modular Antenna/Detector Array for KSTAR Operation}

In Figure 12, an array box configuration deployed with the proposed modular antenna/detector array is presented. The proposed modular antenna/detector array has been adopted in the KSTAR ECE imaging receiver since the 2021 operation with the above configuration. The beams of the ECE signal (as RF) and the LO signal are radiated perpendicularly and pass through one of two windows, respectively. Each of the two signals are divided and combined by a beam splitter, and the combined RF-LO signal beams in two paths are delivered to the two antenna/detector arrays, respectively.

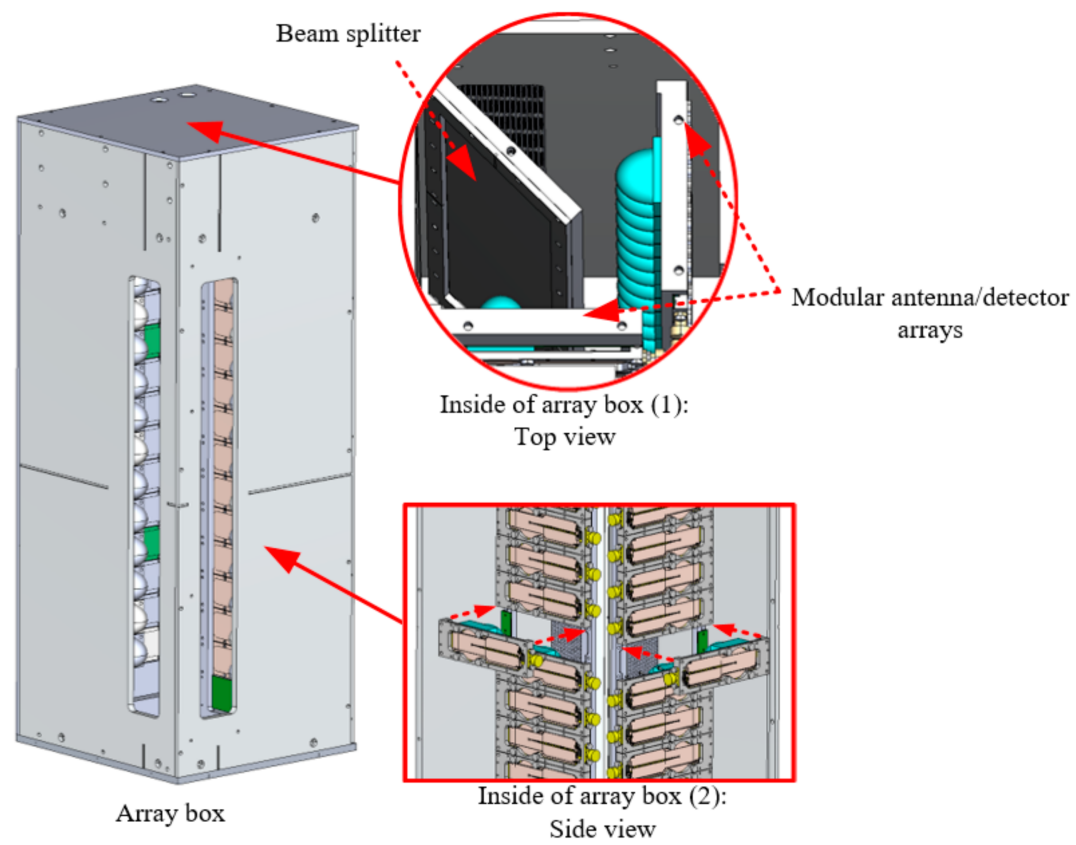

Figure 12. Proposed modular antenna/detector array in an array box.

In case of performance failure due to a single or multiple channel(s), the specific channel module(s) can be easily removed and replaced simply by opening the side metal plate thanks to the modular structure of the proposed array. The specific channel(s) can be individually tuned or improved for performance enhancement. Moreover, when changes in the plasma operation conditions require adjustment of the ECE antenna/detector performance, the proposed antenna/detector modules (including the mini-lens, antenna, and antenna balun) can be easily and independently adjusted or improved due to the modular array setup. Therefore, the proposed modular array can improve the quality of the ECE imaging system by allowing efficient channel maintenance and performance enhancement, thus minimizing the interrupted time for maintenance, and enabling specific system tuning under a variety of tokamak operation conditions.

\section{Conclusions}

The proposed modular antenna/detector array is based on the unit antenna/detector module, which can be independently tuned and installed on the array frame. In case of performance failure due to a specific channel, the corresponding channel module can be easily removed and replaced without interference to the rest of the array, thus minimizing the interrupted time used for maintenance. The unit antenna/detector module consists of an elliptical mini-lens, a dual-dipole antenna with a Schottky diode, an antenna balun, an LNA, and a unit frame. The elliptical mini-lens and dual-dipole antenna with the diode are optimized to have the improved performance in $\mathrm{W}$-band frequencies. For the 
ultra-wideband performance, a CPS-to-MSL transition with optimized design parameters is used. To increase the signal-to-noise ratio, an LNA chip with $15 \mathrm{~dB}$ gain is installed. The fabricated unit antenna/detector modules are tested in a W-band in-house test setup, and consistently provide a gain increase by $10 \sim 20 \mathrm{~dB}$ as compared with the conventional antenna/detector array. Additionally, the proposed modular array allows each unit channel to be efficiently updated under a variety of tokamak operating conditions. For example, combinations of new antenna/detector modules in an array with different performance characteristics can be easily placed and measured without interfering with the rest of the array. This would allow us to successfully measure various plasma instabilities (e.g., tearing mode, edge localized mode, and sawtooth) with changes of plasma conditions.

Author Contributions: Conceptualization, G.H.L., H.K.P. and K.W.K.; methodology, G.H.L.; software, J.S.L., D.H.K. and S.H.N.; validation, G.H.L.; formal analysis, G.H.L. and J.S.L.; investigation, S.H.N., B.C.M. and J.S.L.; resources, G.H.L.; data curation, M.J.K., B.T.K. and T.G.K.; writing-original draft preparation, G.H.L. and K.W.K.; writing—review and editing, K.W.K., J.H.L., W.C.L., G.S.Y., H.C.C., H.K.P. and Y.K.I.; visualization, S.H.N. and M.J.K.; supervision, K.W.K. and Y.K.I.; project administration, K.W.K. and Y.K.I.; funding acquisition, K.W.K. All authors have read and agreed to the published version of the manuscript.

Funding: This research was supported by the National R\&D Program through the National Research Foundation of Korea (NRF) funded by the Ministry of Education, Science and Technology (No. NRF-2019M1A7A1A03088464).

Institutional Review Board Statement: Not applicable.

Informed Consent Statement: Not applicable.

Data Availability Statement: Not applicable.

Conflicts of Interest: The authors declare no conflict of interest.

\section{References}

1. Wesson, J.; Campbell, D.J. Tokamaks, 2nd ed.; Oxford Science Publications: New York, NY, USA, $2004 ;$ pp. 2-7.

2. Yun, G.S.; Lee, W.; Choi, M.J.; Lee, J.; Park, H.K.; Tobias, B.; Domier, C.W.; Luhmann, N.C., Jr.; Donné, A.J.H.; Lee, J.H.; et al. Two-Dimensional Visualization of Growth and Burst of the Edge-Localized Filaments in KSTAR H-Mode Plasmas. Phys. Rev. Lett. 2011, 107, 045004. [CrossRef] [PubMed]

3. Park, H.K.; Luhmann, N.C., Jr.; Donné, A.J.H.; Classen, I.G.J.; Domier, C.W.; Mazzucato, E.; Munsat, T.; van de Pol, M.J.; Xia, Z.; TEXTOR Team. Observation of High-Field-Side Crash and Heat Transfer during Sawtooth Oscillation in Magnetically Confined Plasmas. Phys. Rev. Lett. 2006, 96, 195003. [CrossRef] [PubMed]

4. Yun, G.S.; Park, H.K.; Lee, W.; Choi, M.J.; Choe, G.H.; Park, S.; Bae, Y.S.; Lee, K.D.; Yoon, S.W.; Jeon, Y.M.; et al. Appearance and Dynamics of Helical Flux Tubes under Electron Cyclotron Resonance Heating in the Core of KSTAR Plasmas. Phys. Rev. Lett. 2012, 109, 145003. [CrossRef] [PubMed]

5. Classen, I.G.J.; Westerhof, E.; Domier, C.W.; Donné, A.J.H.; Jaspers, R.J.E.; Luhmann, N.C., Jr.; Park, H.K.; van de Pol, M.J.; Spakman, G.W.; Jakubowski, M.W.; et al. Effect of Heating on the Suppression of Tearing Modes in Tokamaks. Phys. Rev. Lett. 2007, 98, 035001. [CrossRef] [PubMed]

6. Munsat, T.; Domier, C.W.; Kong, X.; Liang, T.; Luhmann, N.C., Jr.; Tobias, B.; Lee, W.; Park, H.K.; Yun, G.S.; Classen, I.G.J.; et al. Electron cyclotron emission imaging in tokamak plasmas. Appl. Opt. 2010, 49, E20-E30. [CrossRef] [PubMed]

7. Zhu, Y.L.; Xie, J.L.; Yu, C.X.; Zhao, Z.L.; Gao, B.X.; Chen, D.X.; Liu, W.D.; Liao, W.; Qu, C.M.; Luo, C.; et al. Millimeter-wave imaging diagnostics systems on the EAST tokamak. Rev. Sci. Instrum. 2016, 87, 11D901. [CrossRef] [PubMed]

8. Wang, J.; Domier, C.W.; Xia, Z.G.; Liang, Y.; Luhmann, N.C., Jr.; Park, H.; Munsat, T.; Mazzucato, E.; van de Pol, M.J.; Classen, I.G.J.; et al. Two-dimensional electron cyclotron emission imaging diagnostic for TEXTOR. Rev. Sci. Instrum. 2004, 75, 3875-3877. [CrossRef]

9. Deng, B.H.; Domier, C.W.; Luhmann, N.C.; Donné, A.J.H.; van de Pol, M.J. Electron cyclotron emission imaging diagnostic on TEXTOR. Rev. Sci. Instrum. 2001, 72, 368-370. [CrossRef]

10. Rowan, W.; Austin, M.; Beno, J.; Ellis, R.; Feder, R.; Ouroua, A.; Patel, A.; Phillips, P. Electron cyclotron emission diagnostic for ITER. Rev. Sci. Instrum. 2010, 81, 10D935. [CrossRef]

11. Yun, G.S.; Lee, W.; Choi, M.J.; Kim, J.B.; Park, H.K.; Domier, C.W.; Tobias, B.; Liang, T.; Kong, X.; Luhmann, N.C., Jr.; et al. Development of KSTAR ECE imaging system for measurement of temperature fluctuations and edge density fluctuations. Rev. Sci. Instrum. 2010, 81, 10D930. [CrossRef] 
12. Janicki, C. Electron temperature measurement from the ECE diagnostics in tokamak plasmas under transient conditions. Nucl. Fusion 1993, 33, 513-516. [CrossRef]

13. Piliya, A.D.; Popov, A.Y. Theory of ECE fluctuations in tokamak plasmas. Plasma Phys. Control. Fusion 2002, 44, 2051-2062. [CrossRef]

14. Yun, G.S.; Lee, W.; Choi, M.J.; Lee, J.; Kim, M.; Leem, J.; Nam, Y.; Choe, G.H.; Park, H.K.; Park, H.; et al. Quasi 3D ECE imaging system for study of MHD instabilities in KSTAR. Rev. Sci. Instrum. 2014, 85, 11D820. [CrossRef]

15. Yang, H.L.; Park, Y.M.; Bae, Y.S.; Kim, H.K.; Kim, K.M.; Lee, K.S.; Bang, E.N.; Kim, H.T.; Lee, H.J.; Kwag, S.W.; et al. Development of KSTAR in-vessel components and heating systems. Fusion Eng. Des. 2011, 86, 588-592. [CrossRef]

16. Lee, W.; Yun, G.S.; Park, H.K.; Nam, Y.B.; Seon, C.R. The conceptual design of an electron cyclotron emission imaging system for studying ITER-like high temperature plasmas. Plasma Phys. Control. Fusion 2014, 56, 95017. [CrossRef]

17. Pan, X.M.; Yang, Z.J.; Ma, X.D.; Zhu, Y.L.; Luhmann, N.C., Jr.; Domier, C.W.; Ruan, B.W.; Zhuang, G. Design of the 2D electron cyclotron emission imaging instrument for the J-TEXT tokamak. Rev. Sci. Instrum. 2016, 87, 11E106. [CrossRef] [PubMed]

18. Nam, Y.B.; Park, H.K.; Lee, W.; Yun, G.S.; Kim, M.; Sabot, R.; Elbeze, D.; Lotte, P.; Shen, J. Compact ECEI system with in-vessel reflective optics for WEST. Rev. Sci. Instrum. 2016, 87, 11E135. [CrossRef] [PubMed]

19. Zhu, Y.; Yu, J.H.; Yu, G.; Ye, Y.; Tobias, B.; Diallo, A.; Kramer, G.; Ren, Y.; Domier, C.W.; Li, X.; et al. W-band system-on-chip electron cyclotron emission imaging system on DIII-D. Rev. Sci. Instrum. 2020, 91, 093504. [CrossRef]

20. Lee, G.H.; Kumar, S.; Choi, H.C.; Kim, K.W. Wideband High-Gain Double-Sided Dielectric Lens Integrated with a Dual-Bowtie Antenna. IEEE Antennas Wirel. Propag. Lett. 2021, 20, 293-297. [CrossRef]

21. Zhang, P.; Domier, C.W.; Liang, T.; Kong, X.; Tobias, B.; Shen, Z.; Luhmann, N.C., Jr.; Park, H.; Classen, I.G.J.; van de Pol, M.J.; et al. The next generation of electron cyclotron emission imaging diagnostics. Rev. Sci. Instrum. 2008, 79, 10F103. [CrossRef]

22. Filipovic, D.F.; Gearhart, S.S.; Rebeiz, G.M. Double-Slot Antennas on Extended Hemispherical and Elliptical Silicon Dielectric Lenses. IEEE Trans. Microw. Theory Tech. 1993, 41, 1738-1749. [CrossRef]

23. Lai, J.; Domier, C.; Luhmann, N.C., Jr. Slot Dual Dipole Antenna with Miniature Elliptical Substrate Lenses for Millimeter-Wave Imaging. Eur. Microw. Conf. 2013, 7, 1751-1754.

24. Lee, G.H.; Mohyuddin, W.; Choi, H.C.; Kim, K.W. Asymmetric Ultra-Wideband Microstrip-to-Coplanar Stripline Transition. IEEE Microw. Wirel. Compon. Lett. 2018, 28, 386-388. [CrossRef]

25. Lee, G.H.; Kumar, S.; Mohyuddin, W.; Choi, H.C.; Kim, K.W. Generalized Design Technique of Ultra-Wideband Transitions for Quasi-TEM Planar Transmission Lines Based on Analytical Models. IEEE Access 2021, 9, 52619-52633. [CrossRef]

26. Lee, G.H.; Mohyuddin, W.; Kumar, S.; Choi, H.C.; Kim, K.W. Compact Wideband Coplanar Stripline-to-Microstrip Line Transition Using a Bended Structure on a Two-Layered Substrate. Electronics 2021, 10, 1272. [CrossRef] 